\title{
A Characterization of Affine Length and Asymptotic Approximation of Convex Discs
}

\author{
Monika Ludwig
}

\begin{abstract}
It is shown that every equi-affine invariant and upper semicontinuous valuation on the space of convex discs is a linear combination of the Euler characteristic, area, and affine length. Asymptotic formulae for approximation of convex discs by polygons are derived, extending results of L. Fejes Tóth from smooth convex discs to general convex discs.
\end{abstract}

1991 AMS subject classification: 52A10, 53A15, 52A27

Keywords: affine length, valuation, Hadwiger's Characterization Theorem, asymptotic approximation

\section{Introduction}

For a convex disc $K$, i.e., a compact convex set in the Euclidean plane $\mathbb{E}^{2}$, the affine length (or the affine perimeter) is defined as

$$
\lambda(K)=\int_{\operatorname{bd} K} \kappa(x)^{\frac{1}{3}} d \sigma(x)
$$

where bd $K$ is the boundary of $K, \kappa(x)$ is the curvature of bd $K$ at $x$, and $\sigma$ is the one-dimensional Hausdorff measure. The notion of affine length was first introduced in the context of affine differential geometry for sufficiently smooth curves (cf. W. Blaschke's monograph [2], a recent survey on planar affine differential geometry is contained in [3]). Since the curvature $\kappa$ is defined almost everywhere and Lebesgue integrable, the same definition can be used for general convex discs. This was pointed out by C. Schütt and E. Werner [18] for $d$-dimensional convex bodies and affine surface area. They showed that this way of defining affine surface area is equivalent to a definition given earlier by K. Leichtweiß [9], who was the first to consider affine surface area for general convex bodies in $d$-dimensional space. In the planar case already L. Fejes Tóth [5] used a notion of affine length defined for general convex discs, which was shown to be equivalent to (1) by K. Leichtweiß [11]. 
Let $\mathcal{K}^{2}$ be the space of convex discs equipped with the topology induced by the Hausdorff metric. $\lambda: \mathcal{K}^{2} \rightarrow \mathbb{R}$ has the following properties:

(i) $\lambda$ is equi-affine invariant, i.e., for every $K \in \mathcal{K}^{2}$

$$
\lambda(\phi(K))=\lambda(K)
$$

for every area preserving affine map $\phi$.

(ii) $\lambda$ is upper semicontinuous, i.e.,

$$
\lambda(K) \geq \limsup _{n \rightarrow \infty} \lambda\left(K_{n}\right)
$$

for every sequence $K_{n}$ of convex discs converging to $K$.

(iii) $\lambda$ is an additive map or a valuation, i.e.,

$$
\lambda(K \cup L)+\lambda(K \cap L)=\lambda(K)+\lambda(L)
$$

for $K, L, K \cup L \in \mathcal{K}^{2}$.

That affine length and affine surface area are upper semicontinuous, follows from a definition of affine surface area given by E. Lutwak [13], which was shown to be equivalent to the other definitions by K. Leichtweiß [10] and G. Dolzmann and D. Hug [4]. It is an immediate consequence of the definition (1) that affine length is a valuation. For the $d$-dimensional case we refer to [17].

We show that these properties - equi-affine invariance, upper semicontinuity, and additivity - characterize affine length $\lambda(\cdot)$, area $\mathrm{A}(\cdot)$, and the Euler characteristic $\chi(\cdot)$.

Theorem 1 Let $\mu: \mathcal{K}^{2} \rightarrow \mathbb{R}$ be an upper (or lower) semicontinuous and equiaffine invariant valuation. Then there are constants $c_{0}, c_{1}$, and $c_{2}$ such that

$$
\mu(K)=c_{0} \chi(K)+c_{1} \mathrm{~A}(K)+c_{2} \lambda(K)
$$

for every $K \in \mathcal{K}^{2}$. If $\mu$ is upper semicontinuous, then $c_{2} \geq 0$, if it is lower semicontinuous, then $c_{2} \leq 0$.

This is a counterpart to H. Hadwiger's characterization theorem [8], p. 221, (see also the surveys [16] and [15]) which states that every continuous and rigid motion invariant valuation on the space of convex bodies in Euclidean $d$-space can be written as a linear combination of the quermass-integrals, i.e., for $d=2$ a linear combination of area, length, and the Euler characteristic.

In $d$-dimensional space affine surface area is an upper semicontinuous and equi-affine valuation. In a subsequent joint paper with $\mathrm{M}$. Reitzner we will extend Theorem 1 and show that every functional defined on the space of convex bodies 
in Euclidean $d$-space which is an upper semicontinuous and equi-affine valuation is a linear combination of volume, affine surface area, and the Euler characteristic.

Affine length and affine surface area are important in problems of asymptotic approximation of convex bodies by polytopes if the distance between the convex body and the polytope is measured by the symmetric difference metric. Here the symmetric difference metric for $K, L \in \mathcal{K}^{2}$ is defined as $\delta(K, L)=A(K \cup L)-$ $\mathrm{A}(K \cap L)$. For information on asymptotic approximation we refer to the surveys [6] and [7].

Let $\mathcal{P}_{n}^{i}(K)$ be the set of convex polygons with at most $n$ vertices that are inscribed in $K$ and define

$$
\delta\left(K, \mathcal{P}_{n}^{i}\right)=\inf \left\{\delta\left(K, P_{n}\right): P_{n} \in \mathcal{P}_{n}^{i}(K)\right\}
$$

i.e., $\delta\left(K, \mathcal{P}_{n}^{i}\right)$ is the distance of $K$ to its best approximating polygons with at most $n$ vertices. In case of circumscribed polygons the analogous notion is $\delta\left(K, \mathcal{P}_{n}^{c}\right)$. For a convex disc $K$ with boundary of differentiability class $\mathcal{C}^{2}$ and with positive curvature, the following asymptotic formulae were given by L. Fejes Tóth [5], p. 43 ,

$$
\delta\left(K, \mathcal{P}_{n}^{i}\right) \sim \frac{1}{12} \lambda(K)^{3} \frac{1}{n^{2}} \quad \text { as } n \rightarrow \infty
$$

and

$$
\delta\left(K, \mathcal{P}_{n}^{c}\right) \sim \frac{1}{24} \lambda(K)^{3} \frac{1}{n^{2}} \text { as } n \rightarrow \infty .
$$

Complete proofs of these results are due to D. McClure and R. Vitale [14], where a description of best approximating polygons is given as well. In Section 4 we show that these results also hold for general convex discs.

In the proof of these asymptotic formulae we make use of two further ways of defining affine length. In Section 3 these definitions are discussed and shown to be equivalent to (1). It is also shown that the equivalence of the definition of affine length used by Fejes Tóth and (1) is a simple consequence of Theorem 1.

We thank I. Bárány for informing us that T. Ódor (unpublished) has obtained independently the characterization of affine length of Theorem 1. We also want to thank Prof. P.M. Gruber, Matthias Reitzner, and Elisabeth Werner for their helpful remarks.

\section{Proof of Theorem 1}

If $\mu$ is lower semicontinuous, then $-\mu$ is upper semicontinuous. Therefore, it suffices to consider upper semicontinuous valuations.

First, we show that proving the following statement implies Theorem 1. 
Let $\mu_{1}: \mathcal{K}^{2} \rightarrow \mathbb{R}$ be an upper semicontinuous and equi-affine invariant valuation with the property that $\mu_{1}(P)=0$ for every polygon $P$. Then there is a constant $c \geq 0$ such that

$$
\mu_{1}(K)=c \lambda(K)
$$

for every $K \in \mathcal{K}^{2}$.

Since $\mu$ is translation invariant, there is a constant $c_{0}$ such that

$$
\mu(K)=c_{0}
$$

for every singleton $K=\{x\}$. We define

$$
\mu_{0}(K)=\mu(K)-c_{0} \chi(K) .
$$

Then $\mu_{0}$ is an upper semicontinuous and equi-affine invariant valuation, which vanishes on singletons.

Next, we show that $\mu_{0}$ is simple, i.e., $\mu_{0}(K)=0$ for every $K$ that is at most one-dimensional. If $K$ is one-dimensional, then it is a closed line segment. By applying an equi-affine invariant transformation $\phi$, we can double its length without changing $\mu_{0}(K)$. Dissecting $\phi(K)$ into two pieces $K_{1}$ and $K_{2}$, both translates of $K$, such that $K_{1} \cap K_{2}$ is a singleton, we obtain

$$
\mu_{0}(K)=\mu_{0}(\phi(K))=\mu_{0}\left(K_{1}\right)+\mu_{0}\left(K_{2}\right)-\mu_{0}\left(K_{1} \cap K_{2}\right)=2 \mu_{0}(K) .
$$

Thus $\mu_{0}(K)=0$ and $\mu_{0}$ is a simple valuation.

Since $\mu_{0}$ is equi-affine invariant, for every triangle $T$ with area $A$ the value of $\mu_{0}(T)$ depends only on $A$, i.e., there is a function $f:[0, \infty) \rightarrow \mathbb{R}$ such that

$$
\mu_{0}(T)=f(A)
$$

By dissecting a triangle into two triangles of area $A_{1}$ and $A_{2}$, respectively, we see that

$$
f\left(A_{1}+A_{2}\right)=f\left(A_{1}\right)+f\left(A_{2}\right)
$$

for $A_{1}, A_{2} \geq 0$. Thus $f$ is a solution of Cauchy's functional equation, and since $\mu_{0}$ and therefore $f$ are upper semicontinuous, we can conclude that there is a constant $c_{1}$ such that

$$
f(A)=c_{1} A
$$

for every $A \geq 0$ (see, e.g., [1]). Taking again into account that $\mu_{0}$ is a simple valuation, we obtain

$$
\mu_{0}(P)=c_{1} \mathrm{~A}(P)
$$

for every polygon $P$. 
We define

$$
\mu_{1}(K)=\mu_{0}(K)-c_{1} \mathrm{~A}(K)
$$

for every $K \in \mathcal{K}^{2}$. Then $\mu_{1}$ is an upper semicontinuous and equi-affine invariant valuation with the property that $\mu_{1}(P)=0$ for every polygon $P$. Thus (4) can be applied and shows that

$$
\mu_{1}(K)=c_{2} \lambda(K)
$$

with a suitable constant $c_{2} \geq 0$, and Theorem 1 follows from (4).

So we have to prove (4). This is done by showing the following result.

For every upper semicontinuous and equi-affine invariant valuation $\nu: \mathcal{K}^{2} \rightarrow \mathbb{R}$ with the property that $\nu(P)=0$ for every polygon $P$, $\nu(K)$ is uniquely determined for every $K \in \mathcal{K}^{2}$ by choosing the value $\nu(B)$ for the solid unit circle $B$.

That (5) implies (4) is seen in the following way. Let $B$ be the solid unit circle and let $\nu(B)$ be given. We have $\nu(B) \geq 0$, since $\nu$ is upper semicontinuous and we can approximate $B$ by polygons $P_{n}$ and consequently,

$$
\nu(B) \geq \limsup _{n \rightarrow \infty} \nu\left(P_{n}\right)=0 .
$$

Therefore we can choose a $c \geq 0$ such that

$$
\nu(B)=c \lambda(B)
$$

Since the affine length $\lambda$ is an upper semicontinuous and equi-affine invariant valuation with the property that $\lambda(P)=0$ for every polygon $P,(5)$ implies for $\lambda$ as well as for $\nu$ that the value for every $K \in \mathcal{K}^{2}$ is uniquely determined by the value for the solid unit circle. Thus (6) implies that

$$
\nu(K)=c \lambda(K)
$$

for every $K \in \mathcal{K}^{2}$, which shows that (4) follows from (5).

So we have to show (5). Let $S \in \mathcal{K}^{2}$ be a sector of the solid unit circle $B$, i.e., the intersection of two closed half-planes with the origin on their boundary and $B$. Since $\nu$ is rotation invariant, $\nu(S)$ depends only on $A=\mathrm{A}(S)$, i.e., there is a function $g:[0, \pi / 2] \rightarrow \mathbb{R}$ such that

$$
\nu(S)=g(A)
$$

Choosing sectors $S_{1}$ and $S_{2}$ such that $S_{1} \cup S_{2} \in \mathcal{K}^{2}$ and $\mathrm{A}\left(S_{1} \cup S_{1}\right)=\mathrm{A}\left(S_{1}\right)+\mathrm{A}\left(S_{2}\right)$ shows that

$$
g\left(A_{1}+A_{2}\right)=g\left(A_{1}\right)+g\left(A_{2}\right)
$$


for $A_{1}, A_{2} \geq 0$ and $A_{1}+A_{2} \leq \pi / 2$. Using (8), we can extend $g$ to a function defined on $[0, \infty)$ that is a solution of Cauchy's functional equation. Since $\nu$ is upper semicontinuous, so is $g$. Thus there is a constant $a$ such that $g(A)=a A$. By $(7), \nu(B)=g(\pi)=a \mathrm{~A}(B)$, which shows that

$$
\nu(S)=\frac{\mathrm{A}(S)}{\mathrm{A}(B)} \nu(B) .
$$

Thus $\nu$ is uniquely determined on all sectors of $B$. A segment of $B$ is the intersection of a closed half-space and $B$. Since $\nu(P)=0$ for polygons $P, \nu$ is also uniquely determined on all segments of $B$, and since $\nu$ is equi-affine invariant, also on equi-affine images of segments.

Let $\mathcal{E}$ be the family of all convex discs which may be dissected into finitely many polygons and segments of $B$ and their equi-affine images. Since $\nu$ is a simple valuation, $\nu$ is uniquely determined on $\mathcal{E}$, and since the polygons belong to $\mathcal{E}, \mathcal{E}$ is dense in $\mathcal{K}^{2}$. Thus the upper semicontinuity of $\nu$ implies that for every $K \in \mathcal{K}^{2}$

$$
\nu(K) \geq \limsup _{k \rightarrow \infty} \nu\left(E_{k}\right)
$$

for every sequence of $E_{k} \in \mathcal{E}$ such that $E_{k} \rightarrow K$.

Showing that for every $K \in \mathcal{K}^{2}$ there are $E_{k} \in \mathcal{E}$ such that $E_{k} \rightarrow K$ and $\nu(K)=\lim \sup _{k \rightarrow \infty} \nu\left(E_{k}\right)$, implies that $\nu$ is uniquely determined on $\mathcal{K}^{2}$ and thus proves (5). So assume on the contrary that there is a $K \in \mathcal{K}^{2}$, necessarily with non-empty interior, such that

$$
\nu(K)>\limsup _{k \rightarrow \infty} \nu\left(E_{k}\right)
$$

for all sequences $E_{k}$ with $E_{k} \in \mathcal{E}$ and $E_{k} \rightarrow K$.

Then there is an $\varepsilon>0$ and a constant $a>0$ such that

$$
\nu(K) \geq \nu(E)+a
$$

for every $E \in \mathcal{E}$ with $\delta(K, E)<\varepsilon$. By the affine isoperimetric inequality (see [13]) $\lambda(E)$ is uniformly bounded for all $E$ with $\delta(K, E)<\varepsilon$. Therefore there is a constant $b>0$ such that

$$
\nu(K) \geq \nu(E)+b \lambda(E)
$$

for every $E \in \mathcal{E}$ with $\delta(K, E)<\varepsilon$. We approximate the solid unit circle by a sequence of convex discs built from suitable pieces of $K$ and show that this leads to a contradiction.

We take an interior point of $K$ and dissect $K$ into small sectors by choosing lines through this point. At every point where such a line intersects the boundary of $K$, we take a support line of $K$. The triangle $T$ bounded by two such support lines for two points, say $x, y$, on bd $K$ together with the chord connecting these 
points is called a support triangle of $K$ and $x, y$ are called its endpoints. In such a way we can assign to every sector $S$ a support triangle $T$.

For a given $n$ we can choose lines such that we obtain $m_{n}$ sectors $S_{1}^{(n)}, \ldots, S_{m_{n}}^{(n)}$ and support triangles $T_{1}^{(n)}, \ldots, T_{m_{n}}^{(n)}$ with the property that

$$
\sum_{i=1}^{m_{n}} \mathrm{~A}\left(T_{i}^{(n)}\right) \leq \frac{1}{n}<\varepsilon .
$$

For every $T_{i}^{(n)}$ with non-empty interior, there is a unique arc of the unit ellipse which touches the two sides of $T_{i}^{(n)}$ which are given by the support lines of $K$. We denote by $E_{i}^{(n)}$ the convex disc bounded by this arc of an ellipse and the chord connecting the endpoints of the support triangle $T_{i}^{(n)}$. In the case that $T_{i}^{(n)}$ has empty interior, let $E_{i}^{(n)}=T_{i}^{(n)}$.

We define $E_{n}$ as the union of $E_{i}^{(n)}$ for $i=1, \ldots, m_{n}$ and $K \backslash \bigcup_{i=1}^{m_{n}} T_{i}^{(n)} . E_{n}$ is a convex disc and $E_{n} \in \mathcal{E}$ and because of $(10), \delta\left(K, E_{n}\right)<\varepsilon$. Since $\nu$ and $\lambda$ vanish on polygons, (9) therefore implies that

$\nu(K)=\sum_{i=1}^{m_{n}} \nu\left(K \cap T_{i}^{(n)}\right) \geq \sum_{i=1}^{m_{n}}\left(\nu\left(E_{n} \cap T_{i}^{(n)}\right)+b \lambda\left(E_{n} \cap T_{i}^{(n)}\right)\right)=\nu\left(E_{n}\right)+b \lambda\left(E_{n}\right)$.

Consequently, there is a $T_{i}^{(n)}$ with non-empty interior such that

$$
\nu\left(K \cap T_{i}^{(n)}\right) \geq \nu\left(E_{n} \cap T_{i}^{(n)}\right)+b \lambda\left(E_{n} \cap T_{i}^{(n)}\right) .
$$

We take an equi-affine transformation $\phi^{(n)}$ which transforms this $T_{i}^{(n)}$ into a support triangle $T^{(n)}$ of the solid unit circle $B$, and denote by $K^{(n)}$ and $B^{(n)}$ the images under $\phi^{(n)}$ of $K \cap T_{i}^{(n)}$ and $E_{n} \cap T_{i}^{(n)}$, respectively. Then by (11) and the equi-affine invariance of $\nu$, we have

$$
\nu\left(K^{(n)}\right) \geq \nu\left(B^{(n)}\right)+b \lambda\left(B^{(n)}\right) .
$$

Let $l_{n}$ be the largest integer such that there are rotations $\psi_{1}, \ldots, \psi_{l_{n}}$ such that $\psi_{1}\left(T^{(n)}\right), \ldots, \psi_{l_{n}}\left(T^{(n)}\right)$ are non-overlapping support triangles of $B$. Since for a sector with an angle $2 \alpha$ at the origin the area of a support triangle to $B$ is

$$
\sin ^{2} \alpha \tan \alpha
$$

we have

$$
\sin ^{2}\left(\frac{\pi}{l_{n}+1}\right) \tan \left(\frac{\pi}{l_{n}+1}\right) \leq \mathrm{A}\left(T^{(n)}\right) \leq \sin ^{2}\left(\frac{\pi}{l_{n}}\right) \tan \left(\frac{\pi}{l_{n}}\right) .
$$

We construct convex discs $K_{n}$ and $B_{n}$ by taking the union of $\psi_{i}\left(K^{(n)}\right)$ and $\psi_{i}\left(B^{(n)}\right)$, respectively, for $i=1, \ldots, l_{n}$ and of $B \backslash \bigcup_{i=1}^{l_{n}} \psi_{i}\left(T^{(n)}\right)$. Then

$$
B_{n}=B
$$


and (12) implies that

$$
\nu\left(K_{n}\right) \geq \nu\left(B_{n}\right)+\frac{b}{2} \lambda\left(B_{n}\right)=\nu(B)+\frac{b}{2} \lambda(B)
$$

for $n$ sufficiently large. Since $\delta\left(K_{n}, B\right) \leq l_{n} \mathrm{~A}\left(T^{(n)}\right)$, we obtain by (14)

$$
K_{n} \rightarrow B
$$

as $n \rightarrow \infty$. Thus by the upper semicontinuity of $\nu$, by (16) and (15), we obtain

$$
\nu(B) \geq \limsup _{n \rightarrow \infty} \nu\left(K_{n}\right) \geq \limsup _{n \rightarrow \infty}\left(\nu\left(B_{n}\right)+\frac{b}{2} \lambda\left(B_{n}\right)\right)=\nu(B)+\frac{b}{2} \lambda(B) .
$$

This is a contradiction, since $b>0$ and $\lambda(B)>0$. Thus (5) holds and the proof of the theorem is complete.

\section{Some further definitions of affine length}

We call a closed triangle $T=T(x, y)$ a support triangle of a convex disc $K$ with endpoints $x$ and $y$, if $x, y \in$ bd $K$ and $T$ is bounded by support lines to $K$ at $x$ and $y$ and the chord connecting $x$ and $y$. W. Blaschke [2], p. 10, gave the following definition of affine length $\lambda_{1}(K)$. Choose subdivisions $x_{1}, \ldots, x_{n}, x_{n+1}=x_{1}$ of bd $K$ and support triangles $T_{1}, \ldots, T_{n}$ such that $T_{i}=T\left(x_{i}, x_{i+1}\right)$. Then

$$
\lambda_{1}(K)=\lim \sum_{i=1}^{n} \sqrt[3]{8 \mathrm{~A}\left(T_{i}\right)}
$$

where the limit is taken over a sequence of subdivisions with $\max _{i=1, \ldots, n} \mathrm{~A}\left(T_{i}\right) \rightarrow 0$. For smooth convex discs Blaschke showed that this limit always exists and that $\lambda_{1}(K)=\lambda(K)$.

If we choose in a support triangle $T(x, z)$ of $K$ a further subdivision point $y$, we obtain support triangles $T(x, y)$ and $T(y, z)$ and the following elementary anti-triangle inequality holds

$$
\sqrt[3]{8 \mathrm{~A}(T(x, z))} \geq \sqrt[3]{8 \mathrm{~A}(T(x, y))}+\sqrt[3]{8 \mathrm{~A}(T(y, z))}
$$

(cf. [2], p. 38, or [3]). This implies that $\sum_{i=1}^{n} \sqrt[3]{8 \mathrm{~A}\left(T_{i}\right)}$ decreases as the subdivision is refined. Consequently, the limit in (17) exists and is independent of the sequence of subdivisions chosen and

$$
\lambda_{1}(K)=\inf \sum_{i=1}^{n} \sqrt[3]{8 \mathrm{~A}\left(T_{i}\right)}
$$

where the infimum is taken over all subdivisions of bd $K$. Thus $\lambda_{1}$ is well defined and K. Leichtweiß [11] proved that $\lambda_{1}(K)=\lambda(K)$ for every convex disc $K$. We show that this is a simple consequence of Theorem 1 . 
Theorem 2 For every $K \in \mathcal{K}^{2}$

$$
\lambda_{1}(K)=\lambda(K)
$$

Proof. By (18) $\lambda_{1}$ is an equi-affine invariant functional defined for every $K \in \mathcal{K}^{2}$ and $\lambda_{1}(K)=0$ for every $K$ which is at most one-dimensional.

For a given $K_{0} \in \mathcal{K}^{2}$ we show that $\lambda_{1}$ is upper semicontinuous at $K_{0}$. If $K_{0}$ has empty interior, this follows immediately from the definition. So let $K_{0}$ have non-empty interior and let $x$ be an interior point of $K$. For a given $n$ divide the plane into $n$ congruent sectors by choosing $n$ rays emanating from $x$. For a $K \in \mathcal{K}^{2}$ such that $x$ is an interior point of $K$, denote the intersection points of these rays with bd $K$ by $x_{1}, \ldots, x_{n+1}=x_{1}$ and set

$$
\mu_{n}(K)=\sum_{i=1}^{n} \sqrt[3]{8 \mathrm{~A}\left(T_{i}\right)}
$$

where $T_{i}$ is that support triangle of $K$ with endpoints $x_{i}$ and $x_{i+1}$ which has minimum area. Then $\mu_{n}$ is continuous at $K_{0}$ and

$$
\lambda_{1}\left(K_{0}\right)=\inf _{n} \mu_{n}\left(K_{0}\right)
$$

Thus $\lambda_{1}$ is the infimum of continuous functionals and therefore upper semicontinuous.

Since $\max _{i=1, \ldots, n} \mathrm{~A}\left(T_{i}\right) \rightarrow 0$, we have for every line $H$

$$
\lambda_{1}(K)=\lambda_{1}\left(K \cap H^{+}\right)+\lambda_{1}\left(K \cap H^{-}\right)
$$

where $H^{+}$and $H^{-}$are the closed half-spaces bounded by $H$. This implies that $\lambda_{1}$ is a valuation. Thus we can apply Theorem 1 , and since $\lambda_{1}$ is simple, we obtain

$$
\lambda_{1}(K)=c \lambda(K)
$$

with a constant $c \geq 0$. By taking a subdivision of the unit circle with equi-distant points, we obtain by (13) that $c=1$.

A further definition of affine length is given in Blaschke's book [2], p. 11. As above choose subdivisions of bd $K$ and support triangles $T_{1}, \ldots, T_{n}$. Then

$$
\lambda_{2}(K)=\lim \sum_{i=1}^{n} \sqrt[3]{12 \mathrm{~A}\left(K \cap T_{i}\right)}
$$

where the limit is taken over a sequence of subdivisions with $\max _{i=1, \ldots, n} \mathrm{~A}\left(T_{i}\right) \rightarrow 0$. Blaschke again showed that this limit exists and is equal to the other definitions of affine length in the case of smooth convex discs. Similarly, affine length can be defined as

$$
\lambda_{3}(K)=\lim \sum_{i=1}^{n} \sqrt[3]{24 \mathrm{~A}\left(T_{i} \backslash K\right)}
$$


where the limit is taken over a sequence of subdivisions with $\max _{i=1, \ldots, n} \mathrm{~A}\left(T_{i}\right) \rightarrow 0$.

To show that $\lambda_{2}$ and $\lambda_{3}$ are well defined and equal to $\lambda$ in the case of general convex discs, we make use of a result by L. Fejes Tóth [5], p. 46. To formulate it, we need the function $\Phi(q), 0<q<1$, defined in the following way. For a given triangle $T=T(x, y)$ and a given value $q$, there is a unique convex $\operatorname{disc} C$ bounded by an arc of a conic and the chord connecting $x$ and $y$ such that $T$ is a support triangle of $C$ and $q=\mathrm{A}(C \cap T) / \mathrm{A}(T)$. Define

$$
\Phi(q)=\lambda(C \cap T) .
$$

For $q=\frac{2}{3}, C$ is bounded by an arc of a parabola and

$$
\Phi\left(\frac{2}{3}\right)=\sqrt[3]{8 \mathrm{~A}(T)}
$$

for $0<q<\frac{2}{3}, C$ is bounded by an arc of an ellipse and for $\frac{2}{3}<q<1$, by an arc of an hyperbola. Elementary calculations show that $\Phi$ is continuous and strictly increasing for $0<q<\frac{2}{3}$ and strictly decreasing for $\frac{2}{3}<q<1$ (cf. [5], p. 45). Fejes Tóth's result states that for every convex disc $K$ and every support triangle $T$ of $K$,

$$
\lambda(K \cap T) \leq \Phi\left(\frac{\mathrm{A}(K \cap T)}{\mathrm{A}(T)}\right) .
$$

Theorem 3 For every $K \in \mathcal{K}^{2}$

$$
\lambda_{2}(K)=\lambda_{3}(K)=\lambda(K) .
$$

Proof. For a given $K \in \mathcal{K}^{2}$, we show that $\lambda_{2}(K)=\lambda(K) . \lambda_{3}(K)=\lambda(K)$ can be obtained in a similar way.

Let $\varepsilon>0$ be chosen and choose a sequence of subdivisions and support triangles such that

$$
\lambda_{2}^{\prime}(K)=\lim \sum_{i=1}^{n} \sqrt[3]{12 \mathrm{~A}\left(K \cap T_{i}\right)}
$$

exists. For $\max _{i=1, \ldots, n} \mathrm{~A}\left(T_{i}\right)$ sufficiently small, we therefore have

$$
\sum_{i=1}^{n} \sqrt[3]{12 \mathrm{~A}\left(K \cap T_{i}\right)}-\varepsilon \leq \lambda_{2}^{\prime}(K) \leq \sum_{i=1}^{n} \sqrt[3]{12 \mathrm{~A}\left(K \cap T_{i}\right)}+\varepsilon
$$

and by Theorem 2

$$
\sum_{i=1}^{n} \sqrt[3]{8 \mathrm{~A}\left(T_{i}\right)}-\varepsilon^{2} \leq \lambda(K)
$$

Since $\Phi$ attains its maximum at $\frac{2}{3}$, it follows from (20) and (19) that

$$
\lambda\left(K \cap T_{i}\right) \leq \sqrt[3]{8 \mathrm{~A}\left(T_{i}\right)}
$$


for $i=1, \ldots, n$. Let the triangles be numbered in such a way that

$$
\lambda\left(K \cap T_{i}\right) \geq(1-\varepsilon) \sqrt[3]{8 \mathrm{~A}\left(T_{i}\right)}
$$

for $i=1, \ldots, m$ and

$$
\lambda\left(K \cap T_{i}\right)<(1-\varepsilon) \sqrt[3]{8 \mathrm{~A}\left(T_{i}\right)}
$$

for $i=m+1, \ldots, n$.

Then by (19), (24), and (20) and since $\Phi$ attains its maximum at $\frac{2}{3}$, we have for $i=1, \ldots, m$

$$
(1-\varepsilon) \Phi\left(\frac{2}{3}\right) \leq \lambda\left(K \cap T_{i}\right) \leq \Phi\left(\frac{\mathrm{A}\left(K \cap T_{i}\right)}{\mathrm{A}\left(T_{i}\right)}\right) \leq \Phi\left(\frac{2}{3}\right)
$$

Since $\Phi$ is continuous, this implies that there is a continuous function $h(\varepsilon)$ with

$$
\lim _{\varepsilon \rightarrow 0} h(\varepsilon)=1
$$

such that

$$
\frac{1}{h(\varepsilon)} \sqrt[3]{2 \mathrm{~A}\left(T_{i}\right)} \leq \sqrt[3]{3 \mathrm{~A}\left(K \cap T_{i}\right)} \leq h(\varepsilon) \sqrt[3]{2 \mathrm{~A}\left(T_{i}\right)}
$$

for $i=1, \ldots, m$.

By (22), (23), and (25) we have

$$
\begin{aligned}
\sum_{i=1}^{n} \sqrt[3]{8 \mathrm{~A}\left(T_{i}\right)}-\varepsilon^{2} \leq \lambda(K) & =\sum_{i=1}^{m} \lambda\left(K \cap T_{i}\right)+\sum_{i=m+1}^{n} \lambda\left(K \cap T_{i}\right) \\
& \leq \sum_{i=1}^{n} \sqrt[3]{8 \mathrm{~A}\left(T_{i}\right)}-\varepsilon \sum_{i=m+1}^{n} \sqrt[3]{8 \mathrm{~A}\left(T_{i}\right)}
\end{aligned}
$$

Consequently,

$$
\sum_{i=m+1}^{n} \sqrt[3]{8 \mathrm{~A}\left(T_{i}\right)}<\varepsilon .
$$

Combing (21), (27), (28), and (22) shows that

$$
\begin{aligned}
\lambda_{2}^{\prime}(K) & \leq \sum_{i=1}^{m} \sqrt[3]{12 \mathrm{~A}\left(K \cap T_{i}\right)}+\sum_{i=m+1}^{n} \sqrt[3]{12 \mathrm{~A}\left(K \cap T_{i}\right)}+\varepsilon \\
& \leq h(\varepsilon) \sum_{i=1}^{m} \sqrt[3]{8 \mathrm{~A}\left(T_{i}\right)}+\sqrt[3]{\frac{3}{2}} \sum_{i=m+1}^{n} \sqrt[3]{8 \mathrm{~A}\left(T_{i}\right)}+\varepsilon \\
& \leq h(\varepsilon)\left(\lambda(K)+\varepsilon^{2}\right)+\sqrt[3]{\frac{3}{2}} \varepsilon+\varepsilon
\end{aligned}
$$


On the other hand, by (23), (27), (28) and (21)

$$
\begin{aligned}
\lambda(K) & \leq \sum_{i=1}^{m} \sqrt[3]{8 \mathrm{~A}\left(T_{i}\right)}+\sum_{i=m+1}^{n} \sqrt[3]{8 \mathrm{~A}\left(T_{i}\right)} \\
& \leq h(\varepsilon) \sum_{i=1}^{m} \sqrt[3]{12 \mathrm{~A}\left(K \cap T_{i}\right)}+\varepsilon \\
& \leq h(\varepsilon)\left(\lambda_{2}^{\prime}(K)+\varepsilon\right)+\varepsilon
\end{aligned}
$$

Since $\varepsilon>0$ was arbitrary and by (26), this shows that $\lambda_{2}^{\prime}(K)=\lambda(K)$ and since this hold for every sequence of subdivisions of bd $K$ with $\max _{i=1, \ldots, n} \mathrm{~A}\left(T_{i}\right) \rightarrow 0$, this proves the theorem.

\section{Asymptotic approximation of general convex discs}

In the following theorem we show that the asymptotic formulae (2) and (3) also hold for general convex discs. This is done by using Theorem 3. Another way to prove this result would be to first show that

$$
\lim _{n \rightarrow \infty} n^{2} \delta\left(K, \mathcal{P}_{n}^{i}\right)
$$

exists, to show that the functional defined in that way is a valuation, and to show that it is upper semicontinuous. The asymptotic result then follows by applying Theorem 1 and calculating the value for the unit circle. But since we have no simple proof for the existence of the limit and the semicontinuity of the functional, we do not follow this approach.

Theorem 4 For every $K \in \mathcal{K}^{2}$

$$
\lim _{n \rightarrow \infty} 12 n^{2} \delta\left(K, \mathcal{P}_{n}^{i}\right)=\lambda(K)^{3}
$$

and

$$
\lim _{n \rightarrow \infty} 24 n^{2} \delta\left(K, \mathcal{P}_{n}^{c}\right)=\lambda(K)^{3} .
$$

Proof. We prove (29). (30) can be obtained in a similar way.

Let $\varepsilon>0$ be chosen. First, we show that for $n$ sufficiently large

$$
12 n^{2} \delta\left(K, \mathcal{P}_{n}^{i}\right) \leq \lambda(K)^{3}+\varepsilon .
$$

For a polygon $P_{n}$ with vertices $x_{1}, \ldots, x_{n} \in \mathrm{bd} K, \delta\left(K, P_{n}\right)$ depends continuously on $x_{1}, \ldots, x_{n}$. Thus for a given $n$ it is possible to choose a $P_{n}$ such that

$$
\mathrm{A}\left(K \cap T_{1}\right)=\mathrm{A}\left(K \cap T_{2}\right)=\ldots=\mathrm{A}\left(K \cap T_{n}\right)
$$


where $T_{i}=T\left(x_{i}, x_{i+1}\right)$. Then

$$
\delta\left(K, \mathcal{P}_{n}^{i}\right) \leq \delta\left(K, P_{n}\right)
$$

and by (32)

$$
12 n^{2} \delta\left(K, P_{n}\right)=12 n^{2} \sum_{i=1}^{n} \mathrm{~A}\left(K \cap T_{i}\right)=\left(\sum_{i=1}^{n} \sqrt[3]{12 \mathrm{~A}\left(K \cap T_{i}\right)}\right)^{3} .
$$

Thus by Theorem 3, for $n$ sufficiently large

$$
12 n^{2} \delta\left(K, P_{n}\right) \leq \lambda(K)^{3}+\varepsilon
$$

which proves (31).

To show the corresponding lower estimate, choose polygons $P_{n}$ such that $\delta\left(K, \mathcal{P}_{n}^{i}\right)=\delta\left(K, P_{n}\right)$ and let $T_{1}, \ldots, T_{n}$ be support triangles of $K$ to consecutive vertices of $P_{n}$. Then

$$
\delta\left(K, \mathcal{P}_{n}^{i}\right)=\sum_{i=1}^{n} \mathrm{~A}\left(K \cap T_{i}\right)
$$

and

$$
\max _{i=1, \ldots, n} \mathrm{~A}\left(T_{i}\right) \rightarrow 0
$$

as $n \rightarrow \infty$. Applying Hölder's inequality shows that

$$
12 n^{2} \sum_{i=1}^{n} \mathrm{~A}\left(K \cap T_{i}\right) \geq\left(\sum_{i=1}^{n} \sqrt[3]{12 \mathrm{~A}\left(K \cap T_{i}\right)}\right)^{3} .
$$

By Theorem 3, we therefore obtain

$$
\lambda(K)^{3}-\varepsilon \leq\left(\sum_{i=1}^{n} \sqrt[3]{12 \mathrm{~A}\left(K \cap T_{i}\right)}\right)^{3} \leq 12 n^{2} \delta\left(K, \mathcal{P}_{n}^{i}\right)
$$

for $n$ sufficiently large. Since $\varepsilon$ was arbitrary, this combined with (31) proves the theorem.

It follows from the above proof that sequences of polygons $P_{n}$ which are errorbalancing, i.e., for which (32) holds, are asymptotic best approximating, i.e.,

$$
\lim _{n \rightarrow \infty} n^{2} \delta\left(K, P_{n}\right)=\lim _{n \rightarrow \infty} n^{2} \delta\left(K, \mathcal{P}_{n}^{i}\right),
$$

and the respective result for circumscribed polygons also holds. For the case of smooth convex discs, this was shown in [14]. There it was also shown that for a smooth convex disc $K$, the vertices of any sequence of best approximating inscribed polygons are uniformly distributed in bd $K$ with respect to affine length. For sequences of best approximating circumscribed polygons it is shown there that 
the points where the sides of the polygons touch $K$ are uniformly distributed in bd $K$ with respect to affine length (see also [12]). That this also holds for general convex discs with positive affine length, follows from the next theorem, since the vertices of best approximating inscribed polygons lie on bd $K$ and the sides of best approximating circumscribed polygons touch bd $K$.

Theorem 5 For every $K \in \mathcal{K}^{2}$ with $\lambda(K)>0$ and every sequence of asymptotic best approximating polygons $P_{n} \in \mathcal{P}_{n}^{i}(K)$ or $\mathcal{P}_{n}^{c}(K)$,

$$
\lim _{n \rightarrow \infty} \frac{\left|P_{n} \cap H^{+}\right|}{n}=\frac{\lambda\left(K \cap H^{+}\right)}{\lambda(K)}
$$

where $H^{+}$is a closed half-space and $\left|P_{n} \cap H^{+}\right|$is the number of vertices of $P_{n} \cap H^{+}$.

Proof. We need Hölder's inequality in the following version. For $a_{1}, a_{2}, b_{1}, b_{2}>0$

$$
a_{1}^{-2} b_{1}^{3}+a_{2}^{-2} b_{2}^{3} \geq\left(a_{1}+a_{2}\right)^{-2}\left(b_{1}+b_{2}\right)^{3},
$$

and there is equality if and only if

$$
\frac{a_{1}}{a_{2}}=\frac{b_{1}}{b_{2}}
$$

Let $P_{n} \in \mathcal{P}_{n}^{i}(K)$ be a sequence of asymptotic best approximating polygons. Since $0 \leq\left|P_{n} \cap H^{+}\right| / n \leq 1+1 / n$, we can choose a convergent subsequence such that

$$
\lim _{k \rightarrow \infty} \frac{\left|P_{n_{k}} \cap H^{+}\right|}{n_{k}}=c
$$

for a suitable $c, 0 \leq c \leq 1$. We have

$$
\begin{aligned}
12 n_{k}^{2} \delta\left(K, P_{n_{k}}\right) & = \\
= & \left(\frac{n_{k}}{\left|P_{n_{k}} \cap H^{+}\right|}\right)^{2} 12\left|P_{n_{k}} \cap H^{+}\right|^{2} \delta\left(K \cap H^{+}, P_{n_{k}} \cap H^{+}\right) \\
+ & \left(\frac{n_{k}}{\left|P_{n_{k}} \cap H^{-}\right|}\right)^{2} 12\left|P_{n_{k}} \cap H^{-}\right|^{2} \delta\left(K \cap H^{-}, P_{n_{k}} \cap H^{-}\right),
\end{aligned}
$$

where $H^{-}$is the closed half-space complementary to $H^{+}$. Since $P_{n_{k}}$ is asymptotic best approximating, we obtain by Theorem 4

$$
\lim _{k \rightarrow \infty} 12 n_{k}^{2} \delta\left(K, P_{n_{k}}\right)=\lambda(K)^{3}
$$

and since $P_{n_{k}} \cap H^{+} \in \mathcal{P}_{m_{k}}^{i}\left(K \cap H^{+}\right)$where $m_{k}=\left|P_{n_{k}} \cap H^{+}\right|$, by Theorem 4 


$$
\begin{gathered}
\liminf _{k \rightarrow \infty} 12\left|P_{n_{k}} \cap H^{+}\right|^{2} \delta\left(K \cap H^{+}, P_{n_{k}} \cap H^{+}\right) \geq \\
\lim _{n \rightarrow \infty} 12 m_{k}^{2} \delta\left(K \cap H^{+}, \mathcal{P}_{m_{k}}^{i}\left(K \cap H^{+}\right)\right) \\
=\lambda\left(K \cap H^{+}\right)^{3},
\end{gathered}
$$

and similarly

$$
\liminf _{k \rightarrow \infty} 12\left|P_{n_{k}} \cap H^{-}\right|^{2} \delta\left(K \cap H^{-}, P_{n_{k}} \cap H^{-}\right) \geq \lambda\left(K \cap H^{-}\right)^{3} .
$$

Letting $k \rightarrow \infty$ in (36) shows that $c=0$ or $c=1$, if and only if $\lambda\left(K \cap H^{+}\right)=0$ and $\lambda\left(K \cap H^{-}\right)=0$, respectively, and in these cases the statement of the theorem holds. So let $0<c<1$ and $\lambda\left(K \cap H^{+}\right), \lambda\left(K \cap H^{-}\right)>0$. Then we obtain from (36) by using (37), (35), (38), (39), and (33),

$$
\begin{aligned}
\lambda(K)^{3} & \geq\left(\frac{1}{c}\right)^{2} \lambda\left(K \cap H^{+}\right)^{3}+\left(\frac{1}{1-c}\right)^{2} \lambda\left(K \cap H^{-}\right)^{3} \\
& \geq(c+(1-c))^{-2}\left(\lambda\left(K \cap H^{+}\right)+\lambda\left(K \cap H^{-}\right)\right)^{3} \\
& =\lambda(K)^{3} .
\end{aligned}
$$

Thus there is equality in Hölder's inequality, which implies by (34)

$$
\frac{c}{1-c}=\frac{\lambda\left(K \cap H^{+}\right)}{\lambda\left(K \cap H^{-}\right)}
$$

and therefore

$$
c=\frac{\lambda\left(K \cap H^{+}\right)}{\lambda(K)} .
$$

Since this holds for every sequence $n_{k}$ for which $\left|P_{n_{k}} \cap H^{+}\right| / n_{k}$ converges, this proves the theorem in the case of inscribed polygons. The proof for circumscribed polygons is analogous.

\section{References}

[1] J. Aczel, Lectures on functional equations and their applications, Academic Press, New York, 1966.

[2] W. Blaschke, Differentialgeometrie II, Springer, Berlin, 1923.

[3] E. Calabi, P. Olver, and A. Tannenbaum, Affine geometry, curve flows, and invariant numerical approximation, Adv. Math. 124 (1996), 154-196.

[4] G. Dolzmann and D. Hug, Equality of two representations of extended affine surface area, Arch. Math. 65 (1995), 352-356. 
[5] L. Fejes Tóth, Lagerungen in der Ebene, auf der Kugel und im Raum, 2nd ed., Springer, Berlin, 1972.

[6] P.M. Gruber, Aspects of approximation of convex bodies, Handbook of Convex Geometry A (P.M. Gruber and J. Wills, eds.), North-Holland, Amsterdam, 1993, 319-345.

[7] P.M. Gruber, Comparisons of best and random approximation of convex bodies by polytopes, Suppl. Rend. Circ. Mat. Palermo 50 (1997), 189 -216.

[8] H. Hadwiger, Vorlesungen über Inhalt, Oberfläche und Isoperimetrie, Springer, Berlin, 1957.

[9] K. Leichtweiß, Zur Affinoberfläche konvexer Körper, Manuscripta Math. 56 (1986), 429-464.

[10] K. Leichtweiß, Bemerkungen zur Definition einer erweiterten Affinoberfläche von E. Lutwak, Manuscripta Math. 65 (1989), 181-197.

[11] K. Leichtweiß, On the affine rectification of convex curves, Beitr. Algebra Geom., in print.

[12] M. Ludwig, Asymptotic approximation of convex curves, Arch. Math. 63 (1994), 377-384.

[13] E. Lutwak, Extended affine surface area, Adv. Math. 85 (1991), 39-68.

[14] D. McClure and R. Vitale, Polygonal approximation of plane convex bodies, J. Math. Anal. Appl. 51 (1975), 326-358.

[15] P. McMullen, Valuations and dissections, Handbook of Convex Geometry B (P.M. Gruber and J. Wills, eds.), North-Holland, Amsterdam, 1993, 933990.

[16] P. McMullen and R. Schneider, Valuations on convex bodies, Convexity and its applications (P.M. Gruber and J. Wills, eds.), Birkhäuser, 1983, 170-247.

[17] C. Schütt, On the affine surface area, Proc. Am. Math. Soc. 118 (1993), 1213-1218.

[18] C. Schütt and E. Werner, The convex floating body, Math. Scand. 66 (1990), $275-290$.

Monika Ludwig

Technische Universität Wien

Wiedner Hauptstraße 8-10/1142

A 1040 Wien

mludwig@pop.tuwien.ac.at 